\title{
Prevalência e impactos do bullying na saúde de adolescentes: um relato de experiência
}

\author{
Prevalence and Impact of bullying on an adolescent health: an experience report
}

Prevalencia y Impacto do bullying en la salud de los adolescentes: un relato de experiência

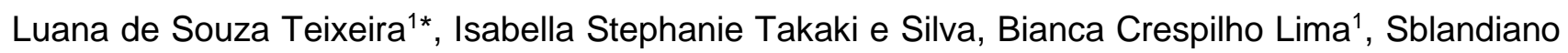
Junqueira Macri ${ }^{1}$, Thais Munhoz Sabadoto ${ }^{1}$, Marinês Ralho'.

\section{RESUMO}

Objetivo: Relatar as experiências advindas de um projeto realizado pelos alunos de medicina, em um território localizado na região sul, de uma cidade do noroeste paulista. Relato de experiência: Este projeto é resultado de práticas desenvolvidas na unidade curricular Prática de Integração Ensino-Serviço-Comunidade (abreviada como PIESC), do curso de medicina, e que objetiva, mediante visita a Unidade Básica de Saúde local, o reconhecimento do território, levantamento dos equipamentos sociais destinados aos adolescentes e a realização de atividades, visando identificar as necessidades de saúde do grupo. Os participantes encontravam-se na faixa etária entre onze e catorze anos e, ao todo, somavam vinte e quatro adolescentes. Desse modo, a partir da vivência, da realização de dinâmicas em grupo e dos resultados obtidos, o tema violência mostrou-se o mais recorrente, tornando-se necessário aprofundamento acerca do tema. Considerações finais: Nesse contexto, entre os componentes do grupo, pôde-se observar a presença do bullying como forma de manifestação da violência psicológica, e sua ocorrência através de comportamentos intimidadores e de agressões verbais. Desta forma, verificou-se o impacto do bullying nos relacionamentos e na saúde dos adolescentes, justificando a importância do desenvolvimento do presente estudo.

Palavras-chave: Bullying, Adolescentes, Escola.

\section{ABSTRACT}

Objective: Report the experiences arising from a project carried out by medical students in a territory located in the southern region of a city in the northwest of São Paulo. Experience report: This project is the result of practices developed in the curricular unit Practice of Teaching-Service-Community Integration (abbreviated as PIESC), of the medical course, and which aims, through a visit to the local Basic Health Unit, to recognize the territory, to survey the social equipment for adolescents and to carry out activities aimed at identifying the health needs of the group. The participants were aged between eleven and fourteen years old and, in all, there were twenty-four adolescents. Thus, from the experience, the performance of group dynamics, and the results obtained, the theme of violence proved to be the most recurrent, making it necessary to deepen the theme. Final considerations: In this context, among the members of the group, it was possible to observe the presence of bullying as a form of manifestation of psychological violence, and its occurrence through intimidating behavior and verbal aggression. Thus, the impact of bullying on teenagers' relationships and health was verified, justifying the importance of developing this study.

Keywords: Bullying, Adolescents, School.

\section{RESUMEN}

Objetivo: Informar las experiencias surgidas de un proyecto realizado por estudiantes de medicina en un territorio ubicado en la región sur de una ciudad del noroeste de São Paulo. Informe de experiencia: Este proyecto es el resultado de prácticas desarrolladas en la unidad curricular Práctica de Integración DocenteServicio-Comunidad (abreviado como PIESC), del curso de Medicina, y que tiene como objetivo, a través de una visita a la Unidad Básica de Salud local, reconocer el territorio, relevamiento de equipamiento social para adolescentes y realización de actividades encaminadas a identificar las necesidades de salud del grupo. Los

\footnotetext{
${ }^{1}$ Centro Universitário de Votuporanga (UNIFEV), Votuporanga - SP.

*E-mail: teixeira.luana.souza@gmail.com
}

SUBMETIDO EM: $8 / 2021$

ACEITO EM: 8/2021

PUBLICADO EM: 10/2021 
participantes tenían entre once y catorce años y, en total, había veinticuatro adolescentes. Así, a partir de la experiencia, la realización de dinámicas de grupo y los resultados obtenidos, el tema violencia resultó ser el más recurrente, por lo que fue necesario profundizar en el tema. Consideraciones finales: En este contexto, entre los integrantes del grupo, se pudo observar la presencia del bullying como forma de manifestación de violencia psicológica, y su ocurrencia a través de conductas intimidatorias y agresiones verbales. Así, se verificó el impacto del bullying en las relaciones y la salud de los adolescentes, justificando la importancia de desarrollar este estudio.

Palabras clave: Bullying, Adolescentes, Escuela.

\section{INTRODUÇÃO}

A adolescência é definida pela Organização Mundial da Saúde (OMS) como o período entre 10 e 19 anos, na qual o indivíduo vivencia mudanças tanto corporais, como o estirão pondero-estatural e 0 surgimento dos caracteres sexuais secundários, mas também cognitivas e no aspecto social, quando há uma ampliação das relações intrapessoais, interpessoais e com o meio em que está inserido (FAIAL LCM, et al., 2016).

Como resultado dessas influências sociais, da assimilação e rejeição das identificações feitas até o momento e de sua percepção do eu como indivíduo, ocorre a consolidação da identidade (ARAUJO GS, et al., 2021). Nesse período, o indivíduo em formação passa grande parte do dia na escola, ocorrendo inevitavelmente um afrouxamento dos vínculos familiares e um fortalecimento dos laços entre seus colegas de turma. Desta forma, há uma necessidade de aceitação e pertencimento com o grupo, o que faz com que muitas vezes o adolescente tente adequar seus gostos, comportamentos e manias em conformidade com seus semelhantes (SECRETARIA DE ESTADO DA EDUCAÇÃO, 2014).

No entanto, devido a imaturidade e a falsa autonomia sobre sua vida, o adolescente pode manifestar tais comportamentos na forma de rebeldia, transgressão e imprudência, estando mais vulnerável a contrair infecções sexualmente transmissíveis (ISTs), ao uso abusivo de álcool e drogas e, não menos importante, a violência (FAIAL LCM, et al., 2016).

Segundo trecho extraído do Relatório Mundial sobre Violência e Saúde, Krug faz referência a definição de violência da OMS, que consiste no uso da força ou poder, de forma intencional, contra si, outras pessoas ou grupos, podendo ocasionar danos psíquicos, lesões, alterações do desenvolvimento ou morte (MERCY JA, et al., 2017). A violência é uma herança histórica, enraizada em todas as camadas sociais, e que aflora cotidianamente nas relações interpessoais, sendo uma questão, sobretudo, social e que se mostra uma constante na vida de grande parcela da população (NJAINE K, et al., 2020). A natureza desse comportamento pode variar entre violência física, sexual, envolvendo privação ou negligência e psicológica (MERCY JA, et al., 2017).

No Brasil, pode-se comprovar, segundo os dados obtidos pelo Sistema de Vigilância de Violência e Acidentes, que a violência atinge todas etárias. No entanto, pode-se destacar a elevada incidência na faixa etária entre 10 e 19 anos, sendo o segundo grupo etário mais ameaçado e, portanto, considerado um problema de saúde pública e de violação dos direitos previstos pelo Estatuto da Criança e do Adolescente (ECA) (BRASIL, 2017).

Dentre os adolescentes, o segundo tipo mais prevalente é a violência psicológica. Segundo a Lei 13.431, de 2017, que altera o ECA, caracteriza-se como violência psicológica qualquer atitude que discrimine, desrespeite ou deprecie a criança ou adolescente, podendo ocorrer na forma de ameaça, humilhação, agressão verbal, ridicularização, indiferença, isolamento, manipulação ou intimidação sistemática, e que podem prejudicá-los em vários aspectos de sua saúde e desenvolvimento psíquico ou emocional (MINISTÉRIO DA SAÚDE, 2017). De acordo com a Lei no 13.185, em vigor desde 2015, essa intimidação sistemática ocorre repetidamente e sem uma motivação aparente, sendo praticada por um único indivíduo ou um grupo, tanto no espaço doméstico como escolar, cujo objetivo é justamente humilhar e amedrontar a vítima (BRASIL, 2015). 
Tal comportamento é possível devido a uma relação desigual de poder entre os personagens, que pode ser consequência de diferença de idade, desenvolvimento físico ou emocional, ou mesmo do maior apoio dos demais estudantes. Sendo os componentes os seguintes: a vítima da violência; o(s) autor(es) das condutas depreciativas e as testemunhas que presenciaram os atos, mas por temerem represálias silenciamse diante destes atos violentos (PIGOZI PL e MACHADO AL, 2015).

Comumente, os autores do bullying são ou foram vítimas de práticas violentas, que possuem condições familiares adversas, sejam estas marcadas pelo excesso de permissividade ou pelo relacionamento afetivo deficitário. Estes tendem a perpetuar o ciclo de violência com aqueles os quais julgam serem mais indefesos, inseguros e pouco sociáveis, quando então sentem prazer ao dominar e ao receber benefícios sociais decorrentes de tal ato (TREVISOL MTC, et al., et al., 2019).

No entanto, tanto os alvos, como os autores e as testemunhas enfrentam diversas consequências comportamentais, emocionais e sociais, que podem se manifestar inicialmente com o desinteresse ou medo de frequentar a escola, alterações do sono e sintomas depressivos, e que a longo prazo podem impactar diretamente nos demais relacionamentos sociais, no desempenho escolar e, principalmente, na saúde física e mental dos adolescentes (SILVA M e SILVA AG, 2018).

Devido ao grande impacto no desenvolvimento escolar desses jovens em processo de formação, cabe ressaltar a importância de professores capacitados que poderão atuar com medidas de identificação, intervenção e prevenção do bullying no contexto escolar, e dessa forma colaborarão com a proteção de crianças e adolescentes. Desse modo, em conjunto com as famílias poderão traçar estratégias para solucionar esse problema social (OLIVEIRA WPS, et al., 2019).

Desta forma, dadas as características, os efeitos e sua alta prevalência, pode-se afirmar que o bullying constitui um grave problema de saúde pública no Brasil (OLIVEIRA WA, et al., 2017). Posto isto, este artigo tem como objetivo relatar a experiência de um grupo alunos do primeiro período do curso de medicina de uma faculdade do noroeste paulista, a qual foi obtida por meio de atividades desenvolvidas na unidade curricular Prática de Integração Ensino-Serviço-Comunidade (PIESC) em um território na região sul do dado município. Por meio destas atividades e do estabelecimento de vínculos, os alunos puderam perceber as necessidades de saúde apresentadas pelo grupo e a recorrência com que o tema violência psicológica foi citado, fazendo-se necessário um estudo mais aprofundado acerca do tema.

\section{RELATO DE EXPERIÊNCIA}

O presente relato de experiência aborda as vivências dos acadêmicos de medicina do primeiro período de uma universidade do interior do estado de São Paulo, obtidas por meio de atividades desenvolvidas na unidade curricular Prática de Integração Ensino-Serviço-Comunidade (PIESC). Essas atividades foram direcionadas à adolescentes, em que por meio de entrevista e dinâmicas lúdicas, percebeu-se a recorrência do tema bullying, o que por sua vez levou à uma reflexão coletiva acerca do tema no grupo em questão.

Nesses encontros, participaram vinte e quatro adolescentes com faixa etária entre onze e catorze anos, em que incluiu dez meninos e catorze meninas. Dessa forma, após uma observação geral do grupo e da criação de vínculo com os participantes, os acadêmicos buscaram levantar a necessidade de saúde em destaque naquele contexto social. Isso foi feito por meio de uma entrevista norteadora para compreender de forma individual cada adolescente, como também analisá-los quando inseridos em sua micro sociedade.

Posteriormente, os alunos de medicina reuniram-se para análise dos dados obtidos por meio da observação e da entrevista. Assim, concluíram que o tema mais pertinente a ser abordado era o bullying, uma vez que tal comportamento foi observado pelos acadêmicos com elevada frequência, como também, pelo relato de experiências vivenciadas pelos próprios adolescentes envolvendo o tema.

O planejamento, desenvolvimento e execução do trabalho foram subsidiados pela Política Nacional de Atenção Básica (PNAB) e pelo Programa de Saúde e do Adolescente e Programa de Saúde na Escola (PSE). Utilizou-se o método observacional que, segundo Gil AC (2008) é um dos mais utilizados nas ciências sociais, podendo ser considerado como o mais primitivo e, por outro lado, pode ser tido como um dos mais modernos, o que possibilita um alto grau de precisão. 
Nesse viés, Prodavov CC e Freitas EC (2013) enfatizam que qualquer investigação em ciências sociais deve se valer, em mais de um momento, de procedimentos observacionais. Esses conceitos foram primordiais para traçar o tema a ser trabalhado, mas também foi de suma importância durante o decorrer das atividades, para assim extrair aprendizados para todos os envolvidos no projeto. O projeto construído pelos acadêmicos de medicina foi organizado em encontros expostos de forma sucinta a seguir:

Quadro 1 - Descrição dos encontros com os adolescentes.

10 Encontro (17/09)
Participantes: 24 Adolescentes
Objetivos: Conhecer o território em que seriam aplicadas as atividades a adolescentes de 11 a 14 anos.
Esse encontro permitiu ainda, conhecer as dependências do local, bem como um diálogo com a
coordenação, possibilitando entender o funcionamento da instituição.
$2^{\circ}$ Encontro (08/10)

Participantes: 24 Adolescentes

Objetivos: Inicialmente, foi aplicado um questionário para obtenção de informações referente aos hábitos de vida e cotidiano dos jovens. Então, com o objetivo de criar vínculos e identificar os assuntos a serem trabalhados com o grupo de adolescentes, no determinado território, foi realizada uma dinâmica.

Atividade: Levava o nome de "Batata Quente", no qual foi utilizado uma bola e, cada participante, ao receber a bola teria que responder perguntas.

\section{Perguntas:}

- Qual é seu nome;

Qual sua idade;

O que gosta de fazer nos tempos livres;

Pratica algum esporte;

Possui animal de estimação;

Onde estuda;

- Qual assunto gostaria que fosse abordado nos encontros;

- Entre outros.

Resultados: De acordo com as respostas adquiridas, identificou-se que a atividade foi eficaz para a criação de vínculo entre o grupo de adolescentes e os integrantes da PIESC. Portanto, no cenário em questão, detectou-se que no próximo encontro o assunto a ser trabalhado seria o bullying.

\section{Participantes: 24 Adolescentes} $3^{\circ}$ Encontro (14/10)

Objetivo: $O$ assunto em foco para elaboração da atividade foi o bullying, pois notou-se a necessidade de promover uma discussão sobre igualdade, empatia e esclarecimento das consequências acerca dos malefícios dessa prática.

Dinâmica: A dinâmica proposta teve o nome de Dinâmica da Inversão, na qual foi pedido para o grupo, que escrevessem em um papel os respectivos 'micos' e destinassem à colegas específicos para realizá-los na presença de todos. No entanto, ao final, foi revelado que tais 'micos' deveriam ser realizados por eles próprios e, com isso, notou-se que muitos adolescentes sentiram constrangidos com o que determinaram para os colegas. Dessa forma, foi possível fomentar a empatia entre eles, já que se colocaram no lugar do outro e notaram a importância de não fazer ao próximo o que não the é conveniente. $O$ encontro foi finalizado com um vídeo que demonstrava a importância da transmissão de boas palavras e atitudes, pois atitudes como essas influenciam positivamente no psicológico de cada indivíduo, alterando o comportamento dos adolescentes na sociedade.

Fonte: Teixeira LS, et al., 2021.

Desse modo, por meio das atividades realizadas durante esses três encontros, os estudantes de medicina buscaram construir de forma gradual uma mudança no convívio social dos adolescentes. Uma vez que, para que pudessem alcançar o desenvolvimento pleno de suas capacidades, era necessário a imersão em um ambiente saudável do ponto de vista da saúde mental, que é peça chave para o domínio do aprendizado. Logo, ao final dos encontros, os acadêmicos presenciaram relatos emocionantes feitos pelos jovens, que demonstraram que a transformação daquele contexto social teria influenciado positivamente na visão em relação a perspectiva de vida de cada adolescente.

Além disso, essas experiências foram de suma relevância para a formação médica dos acadêmicos, visto que refletiram, de forma imersiva, qual seria a melhor maneira para acolher as vulnerabilidades de cada 
indivíduo. Isso porque, puderam constatar a importância de compreender o ser humano como um ser biopsicossocial, uma vez que os aspectos psicológicos e sociais podem interferir negativamente no aspecto físico. Dessa maneira, em um futuro próximo, esses acadêmicos retornarão para a sociedade com o objetivo sólido de exercer uma medicina humanizada.

\section{DISCUSSÃO}

A busca sobre entendimentos acerca do bullying começou há mais de quarenta anos, quando foi definido como 'atos agressivos e intencionais realizados por um grupo ou um indivíduo repetidamente e ao longo do tempo contra uma vítima que não consegue se defender facilmente'. Desse modo, três critérios são relevantes para definir comportamentos agressivos como bullying: (1) repetição, (2) intencionalidade e (3) um desequilíbrio de poder (MENESINI E, et al., 2017).

Nessa mesma perspectiva, o bullying foi analisado tanto globalmente quanto nas 3 categorias: físico, verbal e relacional entre 2006 e 2010/2014 e os resultados mostram estabilidade na sua avaliação e aumento do bullying observado (SÁNCHEZ-QUEIJA I, et al., 2017). Além disso, foi estudado a prevalência desses atos agressivos de acordo com os gêneros. Os resultados revelam que os meninos estão envolvidos em todos os tipos de incidentes de bullying como agressores com muito mais frequência do que as meninas, exceto em casos que envolvem 'falar sobre alguém pelas costas'; nessas situações, as meninas são significativamente mais frequentemente envolvidas como agressores do que os meninos (CARRERAFERNÁNDEZ MV, et al., 2013).

Após essa breve explanação sobre o tema nota-se que o bullying não é um conceito ou comportamento novo e agora está ganhando atenção nacional como uma preocupação crescente de saúde pública. Isso porque, leva a danos físicos e psicológicos de curto e longo prazo, tanto para as vítimas quanto para os agressores (FISHER K, et al., 2017).

Ademais, no contexto escolar foi observado uma maior frequência do bullying na $6^{\mathrm{a}}$ e $8^{\mathrm{a}}$ série do que na 10ª série (ZHANG H, et al., 2017). De acordo, com a percepção dos jovens aqueles que eram 'diferentes', quietos ou impopulares eram mais propensos a serem alvo de agressores. Dessa forma, o bullying continua sendo o principal foco das escolas, embora os programas de prevenção tenham apresentado eficácia limitada (YBARRA ML, et al., 2019).

Nesse sentido em relação aos impactos negativos na saúde física e mental dos estudantes, quadros de ansiedade, baixa autoestima, depressão, automutilação, solidão e suicídio são as situações mais relatadas. Como também afeta o desenvolvimento social, visto que prejudica as relações interpessoais devido a adoção de um estilo passivo de relacionamento ou por apresentar comportamentos agressivos como alternativa de resolução de conflitos (SILVA JL, et al., 2017). Desse modo, entre as medidas para prevenir tais impactos incluem a promoção de inclusão, apoio e segurança a esses adolescentes (GILLESPIE GL, et al., 2018).

Em paralelo com a bibliografia citada acima o nosso projeto obteve como resultado, a observação da ocorrência de ações positivas (cantar, dançar, abraçar o amigo, entre outros), porém também foram notadas diversas práticas de bullying por meio de atitudes que tentavam ridicularizar os colegas durante a realização das tarefas. Desse modo, verificou-se que o bullying trata-se de uma prática recorrente, que envolve comportamentos com atitudes intimidadoras marcadas pela agressão verbal, na maioria dos casos.

Para a consolidação do nosso projeto foram desenvolvidas oficinas voltadas para a educação em saúde, abordando o tema em questão. Uma destas contou com aplicação da "Dinâmica da Inversão", que consistiu em designar uma ação boa ou ruim para que fosse executada pelo colega ao lado, no entanto, no momento da realização, os papéis foram invertidos. Além da dinâmica, foi exibido um vídeo sobre o tema abordado, promovendo o debate acerca do assunto. A partir da exibição do vídeo, os adolescentes puderam refletir a respeito de suas atitudes e vivências marcadas pelo bullying, evidenciando a prevalência deste comportamento no contexto escolar, pois todos os adolescentes afirmaram terem praticado, bem como, terem sido vítimas. 
A percepção dos estudantes do segundo período de medicina diante do primeiro contato com os adolescentes foi de que o bullying, apesar de complexo, pode e deve ser combatido a partir de políticas públicas de atenção à saúde integral do adolescente. Logo, esse trabalho foi de extrema importância para a construção do conhecimento empático e humanizado necessário para a formação de um importante vínculo que será benéfico para os atendimentos futuros.

\section{REFERÊNCIAS}

1. ARAUJO GS, et al. Influência da família na escolha de alunos de graduação em medicina da UEPA - Campus VIII Marabá. Pesquisa, Sociedade e Desenvolvimento, [S. I.], 2021; 10(8): e28210817381.

2. BRASIL. Lei № 13.431, de 4 de abril de 2017. Estabelece o sistema de garantia de direitos da criança e do adolescente vítima ou testemunha de violência e altera a Lei $n^{\circ}$ 8.069, de 13 de julho de 1990 (Estatuto da Criança e do Adolescente). Diário Oficial da União - Seção 1 - 5 de abril de 2017, seção 1, página 1. Disponível em: http://www.planalto.gov.br/ccivil 03/ ato2015-2018/2017/lei/l13431.htm. Acesso em: 31 jul. de 2021

3. BRASIL. Lei № 13.185 , de 6 de novembro de 2015. Institui o Programa de Combate à Intimidação Sistemática (Bullying). Diário Oficial da União, Seção 1, página 1 - 9 de novembro de $2015 . \quad$ Disponível em: http://www.planalto.gov.br/ccivil_03/_ato2015-2018/2015/lei/l13185.htm. Acesso em: 31 jul. de 2021.

4. CARRERA-FERNÁNDEZ MV, et al. Bullying in Spanish Secondary Schools: Gender-Based Differences. The Spanish journal of psychology, 2013; 16: E21.

5. FAIAL LCM, et al. A escola como campo de promoção à saúde na adolescência: revisão literária. Revista PróUniverSUS. 2016; 7(2): 22-29.

6. FISHER K, et al. Bullying: Effects on School-Aged Children, Screening Tools, and Referral Sources. Journal of Community Health Nursing, 2017; 34(4): 171-179.

7. GILLESPIE GL, et al. Review and application of the National Academies of Sciences, Engineering, and Medicine bullying or cyberbullying recommendations for screening and lesbian, gay, bisexual, and transgender youth. Nursing Outlook, 2018; 66(4): 372-378.

8. OLIVEIRA WPS, et al. Levantamento de dados com educadores de uma rede privada de ensino: sondagem dos conhecimentos para identificar, intervir e combater o bullying | Revista Eletrônica Acervo Saúde, 2019; Sup. 31: e1165.

9. MENESINI E, et al. Bullying in schools: the state of knowledge and effective interventions. Psychology, Health \& Medicine, 2017; 22(sup1): 240-253.

10. MERCY JA, et al. Interpersonal Violence: Global Impact and Paths to Prevention. In: Mock CN, Nugent R, Kobusingye O, Smith KR, editors. Injury Prevention and Environmental Health. 3rd ed. Washington (DC): The International Bank for Reconstruction and Development / The World Bank; 2017 Oct 27. Chapter 5. PMID: 30212109. Disponível em: https://www.ncbi.nlm.nih.gov/books/NBK525208/ Acesso em: 22 set. 2021.

11. MINISTÉRIO DA SAÚDE. Secretaria de Vigilância em Saúde. Departamento de Vigilância de Doenças e Agravos Não Transmissíveis e Promoção da Saúde. Viva: Vigilância de Violências e Acidentes: 2013 e 2014 [recurso eletrônico] / Ministério da Saúde, Secretaria de Vigilância em Saúde, Departamento de Vigilância de Doenças e Agravos Não Transmissíveis e Promoção da Saúde. Brasília - DF, Brasil, 2017. 218 p.: il. Disponível em: http://bvsms.saude.gov.br/bvs/publicacoes/viva_2013_2014.pdf. Acesso em: 31 jul. 2021

12. NJAINE K. Impactos da Violência na Saúde [online]. 4th ed. updat. Rio de Janeiro: Coordenação de Desenvolvimento Educacional e Educação a Distância da Escola Nacional de Saúde Pública Sergio Arouca, ENSP, Editora FIOCRUZ, 2020, $448 \mathrm{p}$.

13. OLIVEIRA WA, et al. Saúde do escolar: uma revisão integrativa sobre família e bullying. Ciência \& Saúde Coletiva, 2017; 22: 1553-1564.

14. PIGOZI PL, et al. Bullying na adolescência: visão panorâmica no Brasil. Ciência \& Saúde Coletiva, 2015; 20(11): 3509-3522.

15. PRODAVOV CC, FREITAS EC Metodologia do trabalho científico [recurso eletrônico] : métodos e técnicas da pesquisa e do trabalho acadêmico / Cleber Cristiano Prodanov, Ernani Cesar de Freitas. - 2. ed. - Novo Hamburgo: Feevale, 2013.

16. SÁNCHEZ-QUEIJA I, et al. Trend Analysis of Bullying Victimization Prevalence in Spanish Adolescent Youth at School. The Journal of School Health, 2017; 87(6): 457-464.

17. SECRETARIA DE ESTADO DA EDUCAÇÃO. Superintendência de Educação. Os Desafios da Escola Pública Paranaense na Perspectiva do Professor PDE: Produção Didático-pedagógica, 2014. Curitiba: SEED/PR., Paraná, 2016. V.2. (Cadernos PDE). em: http://www.diaadiaeducacao.pr.gov.br/portals/cadernospde/pdebusca/producoes_pde/2014/2014_uel_ped_pdp_a ndreia_marucci.pdf. Acesso em: 31 jul. de 2021. ISBN 978-85-8015-079-7

18. SILVA JL, et al. Anti-bullying interventions in schools: a systematic literature review. Ciência \& Saúde Coletiva, 2017; 22: 2329-2340.

19. SILVA M, SILVA AG. Professores e Alunos: o engendramento da violência da escola. Educação \& Realidade, 2018; 43: 471-494.

20. TREVISOL MTC, et al. Bullying na adolescência: causas e comportamentos de alunos portugueses e brasileiros1. Revista de Educação PUC-Campinas, 2019; 24(1): 55-72.

21. YBARRA ML, et al. Perceptions of middle school youth about school bullying. J. of Adolescence, 2019; 75: 175-187.

22. ZHANG H, et al. Bullying Behaviors and Psychosocial Adjustment Among School-Aged Children in China. Journal of Interpersonal Violence, 2019; 34(11): 2363-2375. 